\title{
Enhanced Function Structure Applicability through Adaptive Function Templates
}

\author{
By Jan Erik Heller \\ Jörg Feldhusen ${ }^{\dagger}$
}

The concept of function structures is well-established in early phases of engineering design for clarifying product functions. In addition, it forms the basis for the synthesis of solution principles with morphological boxes. Besides benefits, disadvantages remain with its application. First, function structures typically depend on the background their operator. If several persons with diverse backgrounds create function structures, results may be diverging. Second, function structures are non-reversible. It is easy to conceptualize function structures with given products in mind as well as to turn already existing structures into products. However, with unknown context and main functions of existing products there is no method to elaborate unambiguous function structures reversely from those products. Third, function structures are poorly applicable when mixed levels of product embodiment prevail. To overcome those shortages, an improved approach is proposed in this paper. It relies on function templates that are based on already existing function carriers. This is achieved by conceiving building blocks for functions not only consisting of the function description but also of possible function carriers and all subfunctions. For them the same principle of decomposition applies; down to the stage of elementary functions. The conception of pre-created templates helps generating unambiguous structures. By providing context information due to function carrier inclusion, solutions become traceable. Through the templates' adaptive nature, mixed levels get manageable. The paper concludes with discussing the approach regarding the development of multi-technology machine tools. To resolve these challenges, function templates are chosen that are based on the technology chain elements of the manufacturing process.

\section{Introduction}

Innovation is vital for the economic success of enterprises. However, the exclusive concentration on the effectiveness of innovation processes, which

${ }^{*}$ PhD Student, RWTH Aachen University, Germany.

†Professor, RWTH Aachen University, Germany. 
aims at the development of the right products, will not be sufficient. Innovation process efficiency significantly gains importance through changing constraints that result from shorter economic product life cycles as market presence phases shorten. This results in faster and more frequent innovation cycles, high product variance and increased product complexity. Hence, enterprises are forced to turn innovative product ideas into actual products within short time frames offering competitive prices and still fulfilling customers' requirements (Wheelwright \& Clark, 1992). A critical factor of success is the temporal demand of the innovation process. Its importance becomes apparent when looking at the large part development processes usually take of the overall product launch phase (time-to-market) (Pawar, Menon, \& Riedel, 1994). Therefore, a traceable and systematic approach for product development is required today more than ever. A promising approach to handle complexity of products is the use of methods that abstract the problem to a level on which it is manageable. Function modelling seems to be one suitable way to achieve this.

In the first section, this paper presents the general idea of function structures in product development processes. After that, an overview of different approaches is discussed along with their characteristics. Picking up from there, three main challenges for the application of function structures are laid out in detail. The third section introduces a template based model to overcome these shortages. Conclusion and outlook are given in the last section.

\section{Function Structures}

Functions are an adequate means to describe the features that customers desire in products. In many cases, customers directly address the functions they want to be included in the product. Often, they can easily express that they desire a specific function to be present in a product. (E.g. customers could express the wish to be able to change the cutting depth of a lawn-mower. In that case, it is simple to formulate the function change cutting depth.) In contrast to that, it is more difficult or sometimes impossible for the customer to express which physical components have to be designed for a product to be capable of fulfilling that function. E.g. customers may be unaware of how the mechanism for the change of the cutting depth works and which components realise that function. Design engineers often also do not know the details of the system which is going to be developed at the start of the project. Nevertheless, a couple of methods exist (see below) whose purpose is to turn those functions into physical product components or whole products.

Functions typically represent the customers' view of the product. In addition, functions support engineering departments to obtain an overview of the features they have to provide in a product.

This paper uses the expression function in a sense of technical function. Thus, functions itself are describable by inputs, outputs and the transformation of these parameters (Richardson III, 2010). Predominantly, this implies that the 
technical solution (function carrier) may not be known. Other meanings of the expression like economic or aesthetic functions are not addressed in this paper.

\section{Existing Approaches}

Within the last decades, functional modelling of products and the corresponding methods were extensively published.

Bell Laboratories introduced Systems Engineering in the beginning of the 1940s into their product development processes. This approach is centred on the idea of incorporating the functions desired by customers at the earliest possible point in the course of the product development phase (Chestnut, 1967). This methodology is constantly used in enterprises and many software tools exist to support it. The description language SysML in combination with modelling tools from software engineering like UML gained importance in product development processes most recently (Weilkiens, 2007).

Nam Pyo Suh began working on his Axiomatic Design theory in the seventies of last century, which he published two decades later (1990). He presents a consistent methodology for the well-structured design of technical systems. It covers the processes and methods needed for the transformation of a given set of requirements into feasible solutions. Suh pictures four different domains each of them having correlations to their neighbours that are covered by matrices and mathematical operations. The second domain is the so called Functional Domain which addresses the functional requirements of a product.

Koller presents functions as a key part of product development processes (1998). His work is characterised by the fact that he frames out the principle of subdividing or decomposing the so called overall function into subfunctions. Those subfunctions are going to be decomposed again each time by detailing their description and their level of abstraction. This procedure is conducted repetitively until either a technical solution for a function is found or the function is not decomposable. Those functions are called elementary functions. Possible solutions for them are provided by Koller to support engineers with effect catalogues consisting of physical effects that are directly associated with the elementary functions. A basic catalogue is found in (Koller \& Kastrup, 1998).

Pahl and Beitz introduce functions as essential for an organised engineering design process. They unite various approaches from different authors. Particularly the works of Rodenacker, Roth and Krumhauer are to be mentioned. Rodenacker proposes generally valid functions especially intended for the application with binary logic (1991). Roth focusses on the general use of functions and their related structures from a theoretic point of view (2000). Krumhauer concentrates on common functions with special consideration of the types of connections and relations between the function blocks (1974). In their publication, Pahl et al. chose the definitions of Krumhauer (Pahl, Beitz, Feldhusen, \& Grote, 2007).

Ehrlenspiel introduces a marginally changed definition of functions (2007). He argues that working with functions primarily should fulfil the purpose of clarifying the intention of the product before actually beginning 
with the task. He proposes to concentrate on few and simple functions by using accurate descriptions consisting of a noun and a verb. Nevertheless, he reasons, that should this approach turn out to be confusing and not leading to a superior understanding of the actual task because of a large number of functions and complex interrelations between them, it would be appropriate to utilize methods for synthesising function structures like those suggested by Pahl or Koller (Koller, 1998), (Pahl et al., 2007), (Ehrlenspiel et al., 2007).

A methodology that is not directly related to function structures but which is typically used to resolve contradictions that are resulting from them is the TRIZ method of Altshuller (1979). He presents the VePol, i.e. a substance field that is comparable to the physical principles. Compiled lists of physical effects are used to generate solutions analogous to their use within the methodology of Koller.

The function concept is used as well by an approach presented by Umeda et al. The function-behaviour-state model (FBS) enhances the previously presented approaches (Umeda, Takeda, \& Tomiyama, 1990). While it is based on the thoughts of Rodenacker, it extends the field of strict function formulation and decomposition into elementary functions that is required for the application of design catalogues for physical effects. Its use in design processes is exemplarily described by Takeda (1994).

The importance of functions becomes apparent when looking at another aspect that is located further along the product development process. Ulrich presents product architecture as the mapping of functions to the according physical parts of a product (1994). He specifically addresses different types of product architecture, depending on the number of functions fulfilled by specific parts and respectively on the number of parts needed to fulfil a specific function.

\section{Functional Decomposition}

Koller identifies three types of flow: energy, material and signal. Following this, technical systems can be divided into three groups, depending on the type of flow of their overall function or their purpose as machines, apparatuses and devices (Koller, 1998).

To conceive a function structure, first, the purpose of the product currently in development has to be defined. It directly relates to the so called overall function. This overall function features one or more input and output flows. In case a technical solution can be found that fulfils the overall function as well as all other requirements, the engineering task can be considered as completed. However, in most cases, no solution can be retrieved directly. Then, it is appropriate to decompose the overall function into so called subfunctions (Pahl et al., 2007). Numerous techniques can be applied for this decomposition, for example FAST (Wixson, 1999). In most cases, this process is to be considered facile for the first decompositions. However, the more subfunctions exists, it typically gets more difficult to split them up. Koller proposes to decompose all functions down to the level of elementary functions. They are considered generally valid and more important, solution neutral. He created catalogues 
with compilations of physical effects distinguished by the elementary functions they fulfil (Koller \& Kastrup, 1998). The clear advantage of this approach is that it enables engineers to generate principle solutions or product concepts independently from their specific knowledge of physics. Often, it is appropriate to split up the overall function before or during decomposition. A viable distinction is the one into main function and auxiliary function. By definition, only the main functions directly support the purpose (i.e. the overall function). However, several auxiliary functions may be needed in addition to the main function, which add significant complexity to the modelling task. Auxiliary functions can be linked to the main function. For example, when different forms of energy (like heat or other radiation) are required for the actual main conversion of a material, but only electrical energy is available, then their preparation can be considered as auxiliary functions because they do not directly contribute to the main function but still are needed for the product.

Conforming to the three types of flow, the connections between single functions can be considered as energy, material or signal connections. Function boxes are the graphical representations of functions. Usually, a rectangle with a depiction of the function or a description using noun and verb is used. The connections between the functions are displayed with arrows using a different style for each of the three types. The principle of decomposition and the graphical representations of the types of flow are displayed in Figure 1.

Figure 1. Function Structure Representation (acc. to Pahl et al., 2007)

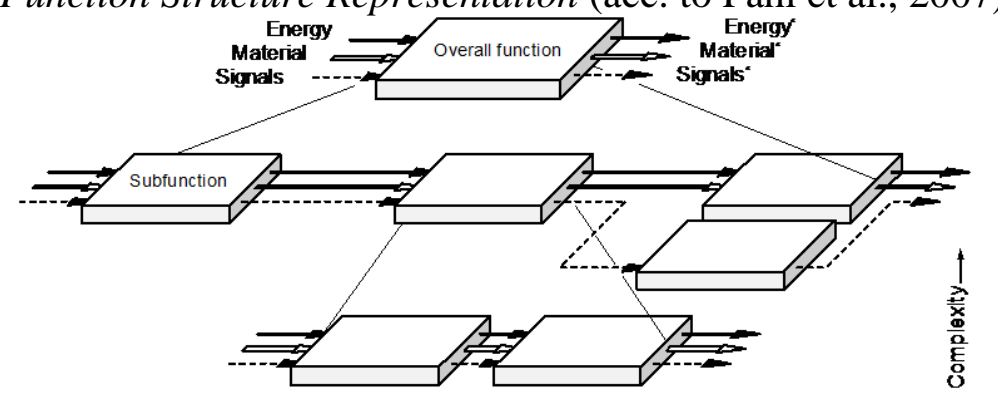

\section{Disadvantages of the Presented Approach}

Despite all advantages and the copious approaches broadly published, several disadvantages with the application, especially for non-academic investigations remain. In the course of a research project, it was the author's task to apply the model of function structures to generate principle solutions in the field of new machine-tools that integrate several manufacturing technologies into single machines. However, for the direct application, several challenges occurred. The next section addresses these disadvantages and discusses the three most important in detail.

\section{Background of the Operator}

When working with function structures, the first challenge is to model the right things in the right way. Although the method of defining the overall 
function first and then subsequently decomposing that function into several subfunctions until the level of elementary functions is reached for each branch of the structure is well-described, it turns out, that two engineers will not necessarily create the same function structure for the same technical system. It is obvious that the individual personal background plays a significant role when preparing function structures. The importance of human influence on engineering tasks in general is covered by Hinsch et al. (2012).

The authors conducted a test where the task was to work out a function structure for a conventional coffee maker. They observed that students, who were not familiar with the process of brewing coffee, in particular achieved results not as useful and accurate as those, who actually were. All students were instructed with the same precise description of the process in text form. It is arguable, that it always will be difficult to create function structures for already existing systems. However, industrial engineering tasks will never consist of completely new problems but always feature subsystems for which solutions already exist. They can either directly be used in the new product, which relativizes the need for a function structure for them or they are going to be undertaken a revision, for which a function structure is desirable. Summarizing, it is obvious, that engineers who are familiar with the subject, will likely formulate more accurate function structures.

Especially when dealing with the development of machine tools, the latter aspects apply, as for example people not being familiar with workpiece handling will likely have difficulties in setting up adequate function structures.

\section{Non-reversibility}

The second challenge is the reversibility of function structures. Typically, it is comparatively easy to generate a function structure for a technical problem. In case all functions are considered thoughtfully and all additional requirements are met, a possible technical solution is reasonably easy to conceive with the help of the methods described above. However, what actually is relatively difficult or even strictly impossible, is the reversed action: Getting from the function structure to a product can be considered feasible while the other way-going from physical representation which means from an actual product back to the function structure-will never be doable in a unique and unambiguous way. An example illustrates this circumstance: If an overall function is to turn electrical energy into light, a possible solution will come to mind immediately: a light bulb. (This is of course with respect to the individual background of the person performing the conclusion. If the light bulb is not known to the individual, this solution would not have come to mind. However, it is assumed, that at least one solution similar to the light bulb would have been inventible with the use of elementary functions and physical effects.) In contrast to that, it will be difficult or even impossible to elaborate the one function structure that was used for the development of the light bulb. While some engineers might possibly create the actual function structure, many will create one that describes functions indeed, but not necessarily those originally intended. For example, a valid solution for the light bulb could be to convert 
electrical energy into head radiation, neglecting the creation of light entirely. This simple example may be debatable; however, the same principle of the direct relation becomes apparent, when looking at machine tool components. E.g. the originally intended function of a cogwheel connection may not be unambiguously named in reverse. The most obvious function would be a change of the torque or the revolution speed. Opposed to that, the cogwheels could have also been intended to be used for an inversion of the revolution direction or even the bridging of a distance between two axes.

Concluding, it can be stated, that function structures are in general nonreversible. Especially for the use within development of machine tools, this is a main disadvantage because many components actually already exist and will be reused in new development projects. For those, no unambiguous function structure can be recreated.

\section{Mixed Levels of Embodiment}

The third challenge results from the combination of the first two and addresses the direct applicability of elementary functions. Mixed levels of embodiment refer to the different levels of abstraction that typically result from the decomposition process. For example, if the decision is made to set up a function structure using elementary functions it is expected to conduct the complete modelling with them. Nevertheless, many physical parts are going to be reused unchanged in new products. Hence, there is no need to perform the function modelling for them with elementary functions as physical effects and principle solutions are not going to be changed, too. In fact, no decomposition is needed for some subfunctions. Moreover, sometimes no adequate and unambiguous formulation for these physical parts is possible in retrospect. Characteristically, the decomposing leads to such mixed levels of embodiment, where existing solutions for some subfunctions can be retrieved whereas for others, the full split up to down to the elementary functions' level is required. Conversely, no suitable method exists to simultaneously use subfunctions as well as elementary functions in the same model while still being able to leverage advantages from catalogues of physical effects aiming at potential product innovation. Thus, a new approach is needed that includes both elementary functions and functions of higher abstraction levels.

Summarizing, function structures are an appropriate means of modelling product ideas and serve as a starting point for physical concept generation. However, as presented, their direct application can be challenging.

\section{Template based Functional Model}

To overcome those shortages and still be able to benefit from the advantages of Koller's method for the creation of function structures, the conventional approach has been extended towards a template based model. In addition to the blocks used for elementary functions, it introduces template 
blocks that can be used to build a homogeneous model. The next section presents the new approach.

\section{Extension of the Conventional Model}

The general procedure for the extended model is shown in Figure 2. Regular rectangles refer to actions or process steps whereas inclined ones depict results. The left side shows the enhanced conventional process while the right side shows the process adapted for the needs of machine tool conceptualization.

Figure 2. Proposed General Procedure for Function Structure Establishment
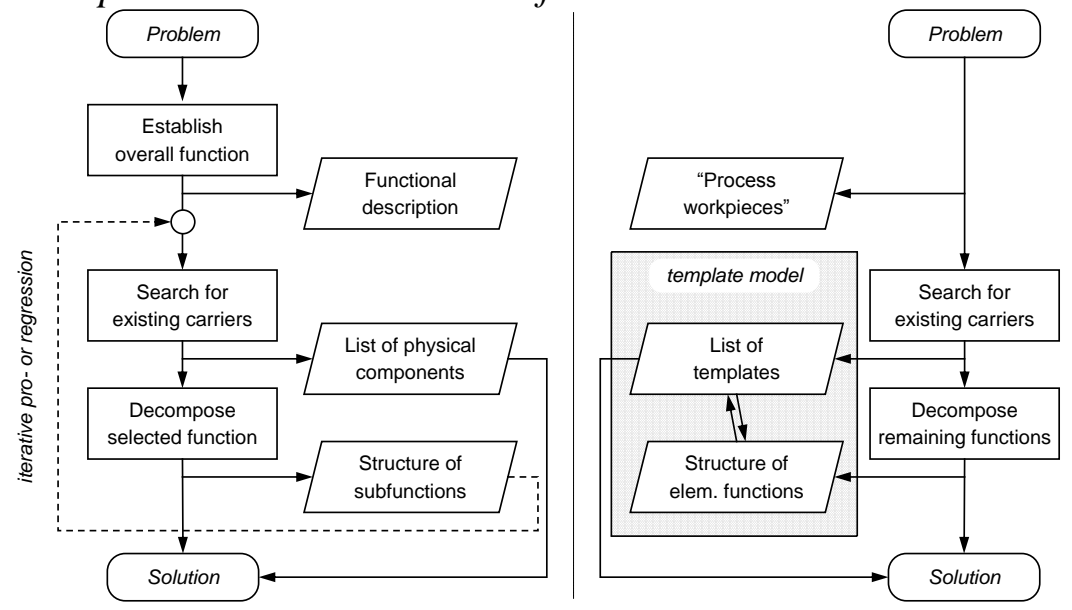

The elaboration of function structures begins with the formulation of the overall function. The result is a first functional description of the product. In the next step, search for existing function carriers begins. As possible carriers are found, the result will be a list of physical components suitable to fulfil that function. If no carriers are found, a decomposition process for the selected function is conducted in the next step which results in a structure consisting of subfunctions. Iteratively, the process is repeated while searching for carriers for those subfunctions until all functions are satisfied. The main result is a possible solution, either consisting of actual physical components or in case of elementary functions consisting of physical effects. For application in the conceptualization of machine tools the first step can be skipped because its result will always be 'process workpieces'. In general, there is no variety in the purpose description of machine tools. Conforming to the conventional procedure the search for function carriers forms the next step. However, it is now carried out based on templates addressing most commonly used functions and their corresponding physical components.

\section{Technology Chains as Input}

Instead of starting from scratch after having formulated the overall function, it is appropriate to begin the modelling process on the basis of already existing objects. In case of the underlying example, the technology chain of the intended machine has been chosen. Technology chains are an established 
means of modelling different manufacturing operations with their dependencies. For the modelling of technology chains, a new kind of flow in addition to the three already known ones is introduced. This flow represents the transformations of the workpiece. As a graphic representation, a thick line with light grey colour and rounded tips is chosen. During the following steps, technology boxes serve as starting points for function structures. They represent single manufacturing processes. Elementary functions and complete subsystems of several functions are going to be assigned to the functional input and output connections of those boxes. Therefore, it is required to obtain a complete definition of all functional interfaces. In addition to intrinsic technological aspects, requirements can be defined. For example, a turning process will need rotational energy to drive the workpiece besides auxiliary functions like lubrication and cooling. The latter require a preparation of the cutting fluid. The main flows can be elaborated and elementary functions from the Koller methodology can be complemented. Exemplarily, the discussed turning process is shown in Figure 3 as a detail from an entire technology chain. Here, the flow of the workpiece is displayed from top to bottom with the main flows of the conventional function structure shown horizontally.

Figure 3. Combination of Function Structure with Technology Box

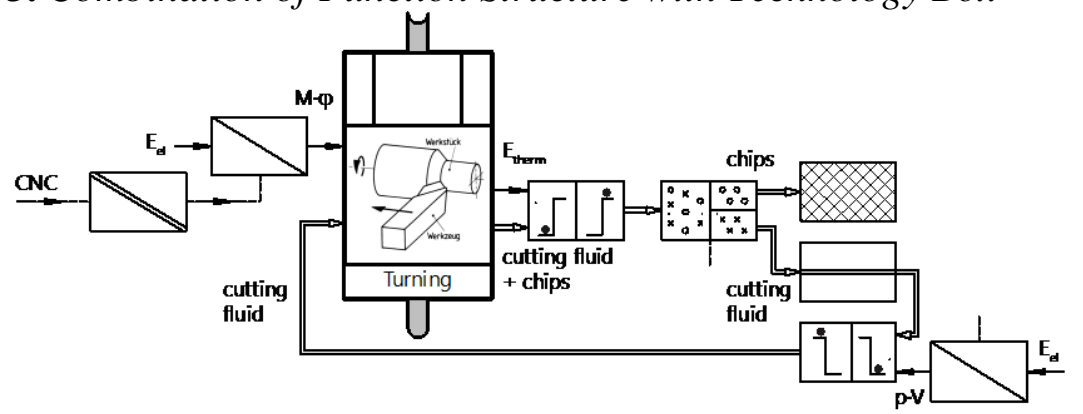

Starting from the left, input values like electrical energy, which is regulated by a $\mathrm{CNC}$ control unit that itself uses $\mathrm{CNC}$ signals as input and the cutting fluid are conducted to the technology box. The output values of the manufacturing process are displayed on the right side of the box. Simplifying, the thermal energy of the process heat and the combined flow of cutting fluid and chips are visualised. In this basic example, the heat is conducted by cutting fluid and chips. After that, the chips are separated from the cutting fluid and stored. With the help of additional electrical energy, the cutting fluid is treated for feeding it into the process again. Advantageous for this approach is the solution neutral modelling of the technology chains, by which all possible combinations and alternatives for different manufacturing opportunities are covered. Figure 4 displays the overall model of a technology chain with an attached comprehensive function structure for the production of an actual part. Focus in this case was not to model various technology chains but to search for substructures that work in a functionally similar way aiming at possible technology integration. Manufacturing is conducted along a serial chain of processes without any alternatives. In contrast to the previous example, the 
workpiece flow is modelled as a continuous line in the technology chain, reaching from the left to the right. Additionally, the manufacturing processes sawing, milling, drilling, hardening, grinding and wire cutting are applied.

Figure 4. Detailed Overall Function Structure with Fully Expanded Templates

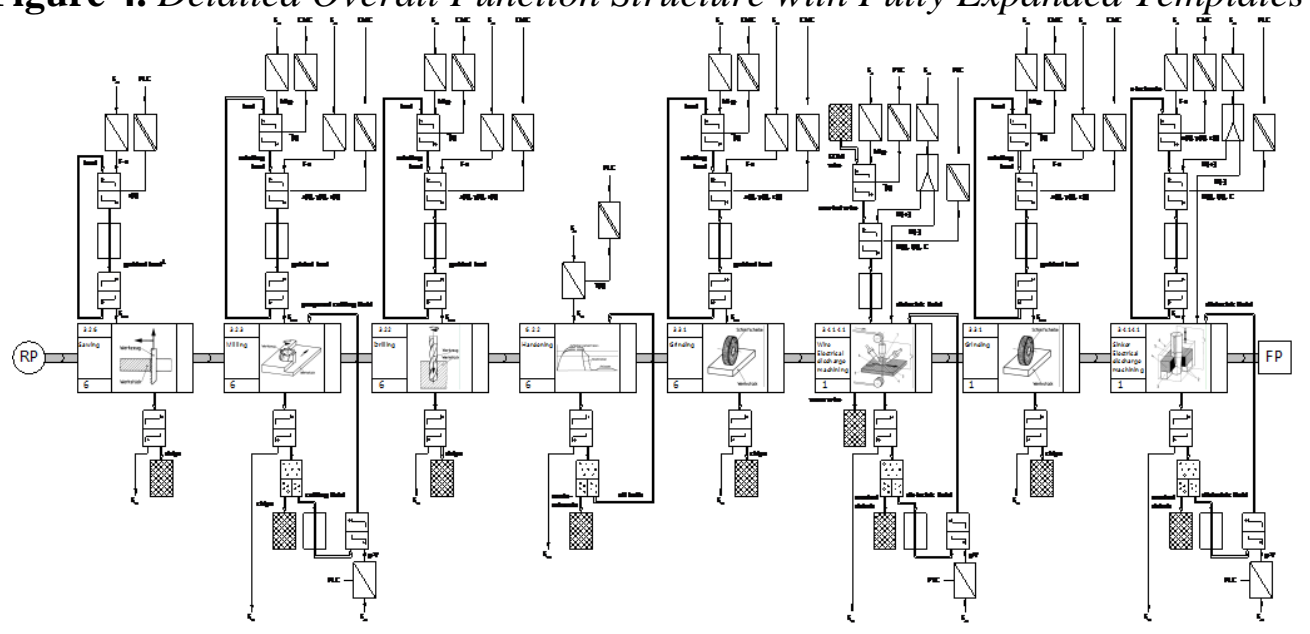

What becomes apparent is that the modelling with elementary functions along the technology chain leads to areas or partial structures consisting of exactly the same functional description. On the one hand, these can be used to leverage integration potential through possible synergetic effects resulting from shared function carriers in hindsight. On the other hand, these groups of functions can be used to establish templates which can be applied in foresight to simplify the modelling process. In addition to that, those then pre-defined templates help to overcome disadvantages resulting from the executors' individual backgrounds by aiding the generation of unambiguous structures.

\section{Adaptive Template Concept}

An exemplary representation of one functional template box for a milling head drive is shown in Figure 5. On the left side, the newly created function box is displayed. As inputs, two forms of electrical energy and two CNC signals are assumed, whereas the output signal is controlled kinetic energy required for the milling operation. In the centre of the figure, the actual underlying function structure composed from conventional elementary functions is shown. This template is directly generated from often used groups of elementary functions which can be noticed when comparing Figures 4 and 5 . However, it is possible to conceive any kind of template by incorporating arbitrary existing groups of elementary functions for which physical representations exist. In Figure 5, corresponding physical components like the electric motor or the linear guides are given as reference for established solution principles. Those components are directly related to the initial functions that lead to their utilisation. Therefore, the addressed reversibility can be achieved by keeping a database of actually used function templates in a product. Summarizing, the presented templates are comprised of: 1.) a template box with textual description and 2.) a graphical representation, 3.) the 
underlying function structure expressed with elementary functions and 4.) a collection of suitable physical components which can be used to realize this group of functions in an actual product.

Figure 5. Exemplary Representation of a Template Box for a Milling Head Drive

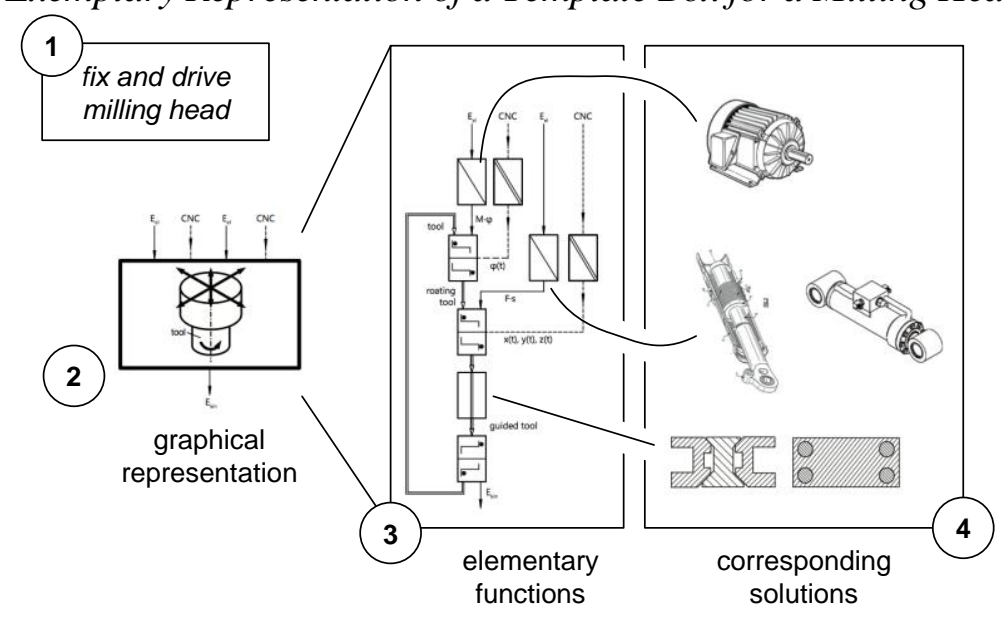

The templates are characterized by their adaptive nature. Operators can directly integrate them in the modelling process and combine them with elementary functions because both feature the same connection types. As the decomposition to the underlying functions is always thinkable the hurdle of mixed embodiment levels becomes manageable: the templates can adopt the prevailing level of concretion according to their environment while still keeping track of corresponding and recognized solutions. Moreover, the recomposition, i.e. the grouping of elementary functions back into a template, is possible with the benefit of directly obtaining possible function carriers.

\section{Conclusion and Outlook}

A new method for conjointly modelling elementary functions as well as functions of a higher abstraction level has been presented. The reasons for this have been discussed and addressed, which leads to better applicability of elementary function structures. Templates have been developed on the basis of the needs of machine tool integration. However, the method is designed openly and thus not restricted to this scope of application.

The topic is subject to current research at RWTH Aachen University. The next step will be to evaluate the efficacy of the proposed approach in educational projects and industrial application. In addition to that, the method will be implemented in a software tool that supports the automatic re- and decomposition of templates as well as programmed identification of possible subfunctions for the use in templates for next generation product development. 


\section{Acknowledgements}

The authors would like to thank the German Research Foundation DFG for the support of the depicted research within the Cluster of Excellence "Integrative Production Technology for High-Wage Countries".

\section{References}

Altshuller, G. S. (1979). Creativity As an Exact Science. Theory of Inventive Problems Solving. Moscow: Sovetskoye Radio.

Chestnut, H. (1967). Systems Engineering Methods. New York, London, Sydney: Wiley.

Ehrlenspiel, K., Kiewert, A., \& Lindemann, U. (2007). Kostengünstig Entwickeln und Konstruieren: Kostenmanagement bei der integrierten Produktentwicklung (6th ed.). Berlin: Springer.

Hinsch, M., Heller, J. E., \& Feldhusen, J. (2012). Improved Application of Design Methodology: Taking Man-Induced Disturbances into Account. In Proceedings of the 14th International Conference on Engineering and Product Design Education E\&PDE. Antwerp.

Koller, R. (1998). Konstruktionslehre für den Maschinenbau: Grundlagen zur Neuund Weiterentwicklung technischer Produkte ( $4^{\text {th }}$ ed). Berlin: Springer.

Koller, R., \& Kastrup, N. (1998). Prinziplosungen zur Konstruktion technischer Produkte (2nd ed.). Berlin: Springer.

Krumhauer, P. (1974). Rechnerunterstützung für die Konzeptphase der Konstruktion (Dissertation). TU Berlin, Berlin.

Pahl, G., Beitz, W., Feldhusen, J., \& Grote, K.-H. (2007). Engineering Design: A Systematic Approach. London: Springer.

Pawar, K. S., Menon, U., \& Riedel, J. C. K. H. (1994). Time to Market: Getting goods to market fast - and first. Integr Manuf Sys, 5(1), 14-22.

Richardson III, J. L. (2010). Incorporating Function Structures into Morphological Charts: A User Study (M. Th.). Clemson Univ, Clemson.

Rodenacker, W. G. (1991). Methodisches Konstruieren: Grundlagen, Methodik, praktische Beispiele (4th ed.). Konstruktionsbücher: Vol. 27. Berlin: Springer.

Roth, K. (2000). Konstruieren mit Konstruktionskatalogen: Konstruktionslehre (3rd ed., Vol. 1). Berlin: Springer.

Suh, N. P. (1990). The principles of design. Oxford series on advanced manufacturing: Vol. 6. New York: Oxford University Press.

Takeda, H., Yoshioka, M., Tomiyama, T., \& Shimomura, Y. (1994). Analysis of Design Processes by Function, Behaviour and Structure. In The Delft Protocols Workshop Conference Proceedings, 261-279. Delft.

Ulrich, K. (1994). The role of product architecture in the manufacturing firm. Research Policy, (24), 419-440.

Umeda, Y., Takeda, H., \& Tomiyama, T. (1990). Function, Behaviour and Structure. In Applications of artificial intelligence in engineering (pp. 177-194). Berlin: Springer.

Weilkiens, T. (2007). Systems engineering with SysML/UML: Modeling, analysis, design. Amsterdam, Boston: Elsevier. 
Wixson, J. R. (1999). Function Analysis and Decomposition Using Function Analysis Systems Technique. In International Council on Systems Engineering Annual Conference INCOSE. 
